\title{
A PATHOLOGICAL FLOW IN THE TORUS ${ }^{1}$
}

\author{
ANATOLE BECK
}

\begin{abstract}
In a recent book (Continuous flows in the plane, Springer Grundlehren, vol. 201, 1974) the author of this article comments that there exist flows in the plane which have the property that some of their orbits have positive planar measure. Indeed, there are flows with the property that the complement of a countable set of orbits has measure 0 . This fact does not appear in the literature, and the author has been asked to provide a proof. He proves instead the existence of a continuous flow in the torus in which the complement of a single orbit has measure 0 . Then, lifting the torus to the plane gives the other example.
\end{abstract}

1. Introduction. In [1], on p. 354, I comment that there exist flows in $\mathbf{R}^{2}$ which contain among their orbits arcs of positive planar measure. In fact, I have shown that there exist flows in the plane which have the property that there are countably many orbits such that the complement of these orbits has measure 0 . This fact does not appear in the literature, and I have been asked to provide a proof. In doing so, I will prove instead the stronger

1.1. Theorem. There exist a flow $\varphi$ in the torus $T^{2}$ and a point $x \in T^{2}$ such that $m\left(T^{2} \backslash \theta_{\varphi}(x)\right)=0$, where $m$ is Haar measure and $\theta_{\varphi}(x)$ is the orbit of $x$.

If we then consider $\mathbf{R}^{2}$ as the universal covering space of $T^{2}$, this will give us a flow in $\mathbf{R}^{2}$ with the required property.

2. Basic notions. A flow $\varphi$ in a space $X$ is a continuous mapping of $\mathbf{R} \times X$ onto $X$ which satisfies the flow equations:

$1^{\circ} \cdot \varphi(0, x)=x, \forall x \in X$,

$2^{\circ} . \varphi(s, \varphi(t, x))=\varphi(s+t, x), \forall x \in X, \forall s, t \in \mathbf{R}$.

For each $x \in X$, the set $\{\varphi(t, x) \mid t \in \mathbf{R}\}$ is called the $\varphi$-orbit of $x$ and is written $\vartheta_{\varphi}(x)$. We do not require any further properties of flows.

We also need the information that for any open, connected set $U$ in $\mathbf{R}^{2}$, there exists an arc $\gamma$ in that set whose 2-dimensional measure is any number less than $m(U)$. This fact is proved in [3], and in [2, pp. $201 \mathrm{ff}$.] for squares, and is easily extended.

3. A first approximation. We will begin with a slightly simpler problem. Instead of having one orbit with all the measure, we will distribute it among countably many. In exchange, we will have every orbit with period 1.

Received by the editors May 27, 1980.

AMS (MOS) subject classifications (1970). Primary 54H20, 28D99, 58 F27.

Key words and phrases. Osgood curve, curve of positive area, irrational flow.

${ }^{1}$ This research was supported by the Wisconsin Alumni Research Foundation. 
3.1. Example. Let $T^{2}$ be the 2-torus obtained from the unit square $Q$ by identifying the sides in the usual way. I shall produce a flow $\varphi$ in $T^{2}$ in which every orbit has period 1 and in which there is a countable number of orbits whose total 2-dimensional measure is 1 .

I will produce a homeomorphism $h$ of $Q$ onto $Q$, and $\varphi$ will be the image under $h^{-1}$ of the flow $\xi$ defined by $\xi(t,(x, y))=(x+t, y)(\bmod 1) . h$ will be defined as the identity on $\partial(Q)$.

To do this, I will first define the concept of a flake. A flake $S$ is a simplyconnected open set in $Q$ bounded by a Jordan curve which is the union of four arcs, which are pairwise disjoint except for four points called corners where two of them meet. Two of these arcs which do not meet are called sides, and are parametrized over the same interval in the opposite senses as one traverses $\partial(S)$. The remaining arcs are called the top and bottom of $S$, and are also parametrized over some common interval, again in the opposite order. I will now define a process which I will call shredding $S$ by $m$ and $n$.

We know that there is a conformal mapping $g$ of $S$ onto a rectangle $R$ which carries the top of $S$ onto the top of $R$, etc. This mapping is unique up to geometric similarity. Let the sides of $S$ be divided into $m$ arcs each by $m-1$ points equally spaces according to the parametrization, and let the top and bottom be similarly divided into $n$ arcs each. We now consider the images of these points in $\partial(R)$, where they are found by continuous extension of $g$. The points need not be equally spaced, but the corresponding points do lie in the same order from left to right or top to bottom. Join these corresponding points by straight line segments to create in $R$ a kind of tipsy graph-paper consisting of $m n$ quadrilaterals. Let the sides of these quadrilaterals be parametrized in such a way that each is a flake, and so that the parametrization agrees with the parametrization of $\partial(R)$ where these meet. The tops, bottoms and sides of the new quadrilaterals correspond to those of $R$. Mapping back by $g^{-1}$, we obtain a decomposition of the flake $S$ into $m n$ flakes. We designate the maximum diameter of these $m n$ flakes as the mesh of the shredding, and note that by the continuity of $g$, the mesh can be made arbitrarily small by choosing $m$ and $n$ large enough. Furthermore, we note that the arcs removed from $S$ by this process are all analytic arcs, and thus have 2 -measure 0 .

We will now define another process, which we call winnowing the flake $S$. We locate the midpoint of each side of $S$, according to its parametrization. We now construct an arc $\gamma$ lying in $S$ which has these midpoints for endpoints and has 2-measure equal to at least half the measure of $S$. Let $\gamma$ be parametrized to correspond to the common parametrization in the top and bottom of $S$. Then $\gamma$ cuts $S$ into two flakes whose total measure is less than half the measure of $S$.

We are now ready to construct the homeomorphism $h$. We choose any $0<\varepsilon<$ 1 , and winnow $Q . h$ is the identity on $\partial(Q)$, and the winnowing arc is mapped onto the line segment joining $\left(0, \frac{1}{2}\right)$ to $\left(1, \frac{1}{2}\right)$, according to its parametrization. Then we shred each of the remaining flakes by $2^{n_{1}-1}$ and $2^{n_{1}}$, where $n_{1}$ is chosen large enough so that the meshes of both shreddings are less than $\varepsilon$. We now have $2^{2 n_{1}}$ flakes, and we define $h$ on their boundaries in the obvious way according to their 
parametrizations so that their images will be the set of all $(x, y) \in Q$ for which $x$ or $y$ is of the form $j \cdot 2^{-n_{1}}$, with $j$ an integer.

In the second step, we winnow each of the $2^{2 n_{1}}$ flakes created in the first step, thus giving us $2 \cdot 2^{2 n_{1}}$ flakes whose total measure is less than $\frac{1}{4}$. We define $h$ on these new arcs in the obvious way, so that their image will consist of all points $(x, y) \in Q$ with $y$ of the form $j \cdot 2^{-n_{1}-1}$, where $j$ is an odd integer. We now shred each of the $2 \cdot 2^{2 n_{1}}$ flakes by $2^{n_{2}-1}$ and $2^{n_{2}}$, with $n_{2}$ chosen so large that all of the $2 \cdot 2^{2 n_{1}}$ shreddings have meshes less than $\varepsilon^{2}$, and we define $h$ on the edges of the resulting flakes so that we now have a mapping onto all the points $(x, y) \in Q$ with $x$ or $y$ of the form $j \cdot 2^{-n_{1}-n_{2}}$.

Continuing in this way, we define the mapping $h$ on a dense set in $Q$, consisting of the edges of all the flakes defined successively. The image of this set will be the set of all $(x, y) \in Q$ where either $x$ or $y$ is a dyadic rational. Since the meshes of the shreddings obtained at the $k$ th step are always less than $\varepsilon^{k}$, we see that $h$ can be extended by continuity to a homeomorphism of $Q$ onto $Q$. Let $H$ be the union of the horizontal line segments joining $(0, a)$ to $(1, a)$ for all $a$ which are dyadic rationals. Then the process of winnowing assigns to $h^{-1}(H)$ at each step a set whose measure exceeds half of the 2-measure not previously so assigned. It follows that $m\left(h^{-1}(H)\right)=1$. Setting $\varphi=h^{-1}[\xi]$, we see that $h^{-1}(H)$ is a set of measure 1 consisting of countably many $\varphi$-orbits. Q.E.A. ${ }^{2}$

4. Proof of Theorem 1.1. We will now modify the previous example by defining a different identification between the points on the left and right sides of the unit square $Q$. The new identification will link all the curves of positive measure in the previous example into a single orbit. That will complete the task.

To begin with, we will choose an irrational number $\alpha \in(0,1)$. We will define "the irrational flow of parameter $\alpha$ " in a slightly different way: for any $(x, y) \in Q$, and any $-x \leqslant t \leqslant 1-x$, we define $\psi(t,(x, y))=(t+x, y)$. Now if we identify $(1, y)$ with $(0, y-\alpha)$ modulo $1, \forall y \in I$; the resulting flow is the familiar irrational flow with parameter $\alpha$, written somewhat differently. Consider the orbit of the point $(0,0)$, which we will cut into the $\operatorname{arcs}[(0, j \alpha),(1, j \alpha)), \forall j \in \mathbf{Z}$. We say that each such arc has $\alpha$-number $j$. Next, we count these arcs from 1 to $\infty$, and call these the $\beta$-numbers of these same arcs. This forms a sequence $\left\{\beta_{i}\right\}$ of arcs. Finally, we count the arcs in Example 3.1 which run from the left edge of the square to the right edge and have positive 2 -measure, and call those the $\gamma$-numbers of those arcs. This forms a sequence $\left\{\gamma_{i}\right\}$.

We will now pair off the arcs of the orbit of $(0,0)$ in the irrational flow of parameter $\alpha$ with the arcs of positive 2-measure in Example 3.1 so as to preserve the order relations among them. First, we pair off $\beta_{1}$ with $\gamma_{1}$ and $\beta_{2}$ with $\gamma_{2}$. Next we choose $\gamma_{3}$ and observe it falls into the region above $\gamma_{1}$ and below $\gamma_{2}$, or the reverse. If the former, then we pair $\gamma_{3}$ with that arc lying above $\beta_{1}$ and below $\beta_{2}$ which has the smallest $\beta$-number. If the reverse, then we pair it with that arc lying above $\beta_{2}$ and below $\beta_{1}$ which has the smallest $\beta$-number.

${ }^{2}$ Quod erat agendum. 
We now continue recursively. Once we have paired $\gamma_{1}, \ldots, \gamma_{n}$ with arcs in the $\alpha$-orbit, we observe that $\gamma_{n+1}$ falls into the space between two of them, and choose for the corresponding arc that portion of the $\alpha$-orbit with the lowest $\beta$-number lying between the corresponding $\beta$-arcs. Thus the order is preserved between the $\gamma$-arcs and the corresponding $\beta$-arcs. Since the $\gamma$-arcs are densely ordered, every $\beta$-arc is eventually chosen. Thus, we have a 1-1 pairing of the $\gamma$-arcs onto the $\beta$-arcs.

Now the identification between endpoints of the $\beta$-orbits induces a corresponding identification between the endpoints of the $\gamma$-arcs, and this can be extended uniquely to a homeomorphism of $\mathbf{R} \bmod 1$ onto $\mathbf{R} \bmod 1$. The identification of $(x, 0)$ with $(x, 1), \forall x \in I$, and $(1, y)$ with $(0, h(y))$ now makes $Q$ into a torus. Q.E.D.

\section{BIBLIOGRAPHY}

1. Anatole Beck, Continuous flows in the plane, Die Grundlehren der Math. Wissenschaften, Band 201, Springer-Verlag, Berlin and New York, 1974.

2. Anatole Beck, M. N. Bleicher and D. W. Crowe, Excursions into mathematics, Worth, New York, 1969.

3. W. F. Osgood, A Jordan curve of positive area [sic], Trans. Amer. Math. Soc. 4 (1903), 107-112.

Department of Mathematics, University of Wisconsin, Madison, Wisconsin 53706 